\title{
Fibre Configurations for Moisture Control: A Vernacular Framework
}

\author{
Miriam Ho \\ University of Waterloo, Canada \\ m5ho@uwaterloo.ca
}

\begin{abstract}
This research describes a theoretical framework for making water-sensitive architectural fabrics based on an examination of the subtle moisture controls in vernacular architecture. It explores a workflow for the articulation of environmental forces in material microstructure and how these can be amplified at an architectural scale. The interaction between moisture and material properties is modeled using physics simulations in Processing, offering a method to manipulate fibre configurations for new construction logics. The research is illustrated through parallel explorations in computationally generated fibre configurations and multi-scalar textile prototypes. The research aims to demonstrate the potentials of technology transfer from traditional knowledge to contemporary material manipulations.
\end{abstract}

Keywords: Vernacular architecture; Simulation; Environment; Textiles.

\section{Introduction}

Environmental design strategies are being rapidly transformed by new computational paradigms in generative algorithms (de Beaurecueil \& Lee, 2008); at the same time, contemporary concerns of climate change and environmental degradation demand a new engagement with material ecologies and cultural practices of material knowledge. In particular, a renewed interest in the millenial traditions of vernacular architecture and local craft has much to teach us. Vernacular building practices are rooted in an intimate relationship to the environment through its direct engagement with locally-found materials, and through specific adaptations to local climatic conditions that result in strategic microclimatic modification. The subtle controls of moisture, temperature, light, and air in vernacular building envelopes allow for human thermal comfort and sustainable resource practices that exist in a delicate balance with the environment.

Hasan Fathy's Natural Energy and Vernacular Architecture, a seminal text in propelling a scientific understanding of traditional building types, suggests that through a careful examination of thermodynamics and material properties, technologies from the past can still be relevant today. Vernacular buildings passively respond to the environment through the mutual interaction of materials and atmospheric matter. Fathy presents a minute study of subtle natural phenomenon such as heat conduction and convection, or moisture condensation and evaporation, illustrating each as the result of dynamic fluctuations in density, porosity, temperature and moisture content. These dynamic interactions can be controlled through the physical properties of building materials.

Algorithmic processes offer architects increasing access to the physics of materials and structures at multiple orders of scale
(Andrasek, 2012). The ability to simulate and visualize subtle environmental phenomenon using mathematically generated environmental force systems and their resultant formations allows us to acquire material knowledge at an increased resolution. There are synergies between the generative logic of computational design algorithms, where forms materialize through simulated information flow, and Fathy's study of the vernacular, where material microstructure has a direct relationship to dynamic environmental flows. These synergies offer potential for the technology transfer of vernacular material intelligence using computational simulation and scalable textile craft processes.

\section{Fabrics for Moisture Control}

The interaction of material microstructure and environmental forces is clearly illustrated in the making of a textile. A fabric is a flexible surface constructed out of interlinked polymers which are assembled using a serial logic. The way in which the fibres are interlinked determine the characteristics of the fabric, including its structure capacity, material behaviour and performance. A fabric is thus an assembled structure or material system that is inherently hierarchical (Eadie \& Ghosh, 2011). Following an algorithmic logic, sets of elements or nested elements are repeated to generate a pattern that determines the fabric's topology. By varying construction algorithms, textiles can be functionally graded, varying in material distribution to suit performance. Textiles are increasingly recognized as highperformance materials, with performance linked to its varied structural capabilities (Warwick, 2005).

Fabrics that are capable of capturing, storing, and distributing atmospheric moisture have a wide range of contemporary applications, from fog harvesting for collect potable water to 
athletic-wear that transports sweat away from the body to smart responsive shape-changing fabrics. The specific configuration of the weave or knit, creating a material structure with varying density, pattern and topology, allows fabrics to perform functions of water collection or transport. In developing fibre configurations for water functions, researchers often use biomimicry as a framework: notable examples include polymer films that mimic the hydrophobic and hydrophilic bumps on the Namib desert beetle for fog collection (Trafton, 2006), double-layered knit fabrics connected by biomimetic branching stitches for better water adsorption (Chen, 2011), and the design of shape-changing surfaces inspired by the cellular composition of the pine cone hygromorph (Scott, 2012).

Vernacular material systems offer a different but related framework for moisture control. Vernacular architectures can also capture, store and distribute ambient moisture for evaporative cooling and water harvesting. Like living organisms, vernacular architectures interact with their local environment by regulating the passage of heat and moisture through their envelopes to create new microclimatic conditions. Vernacular architectures use local materials, organic and inorganic, that are inherently sensitive to air temperature and humidity. These materials are reconfigured with a careful consideration of architectural thermodynamics to benefit from the material's capacity to absorb and release moisture over time.

To begin to create architectural fabrics capable of moisture control, we ask the questions of: how can the articulation of environmental forces in material microstructure be amplified to an architectural scale? What are the relationships between microstructure, pattern and overall topology and how can these be functionally graded for moisture management? The research builds upon existing work on moisture-sensitive biomimetic textiles and extends these experimental research traditions to include factors of porosity, density and time lag, which allow passive moisture regulation in vernacular architecture. The research aims to develop a workflow for creating functionally graded, moisture-sensitive textiles.

\section{Research Context}

\section{Interaction of moisture and materials}

The interplay of materials and moisture at different scales allows material assemblies to be programmed for a wide range of functionalities. At the molecular scale, a water molecule - $\mathrm{H} 2 \mathrm{O}$ has a polar nature, consisting of two positively charged hydrogen atoms and one negatively charged oxygen atom. Water molecules are attracted to each other: the hydrogen of one molecule is attracted to the oxygen of another, causing them to form clusters. As temperatures increase, water clusters gain more energy and will break apart into smaller clusters or evaporate as lone water molecules. Materials are thus capable of being both water-tight and water permeable by interacting with the difference in size between water clusters and lone water molecules (Straube, 2006).

Materials can be hydrophobic (repel water), or hydrophilic (attracts water) and hygroscopic (capable of absorbing water) based on their pore structure. The contact angle of a water droplet and the surface beneath determines whether the material is hydrophobic or hydrophilic, where a contact angle greater than 90 degrees creates a hydrophobic surface, and one less than 90 degrees causes water to spread, creating a hydrophilic surface. Water absorption is governed by the porosity of a material, where larger pore sizes results in smaller spaces for water transport via capillary action, resulting in faster water absorption as well as greater heat conductivity. Porous materials have the tendency to capture and hold water molecules in its pores and capillaries. Once a material is fully saturated (has reached its moisture storage capacity), it will no longer transmit moisture, but will release moisture back into the environment.

The rate at which moisture passes through a material is significant for climactic regulation. By exploiting a material's capacity for moisture storage so that it can absorb and give back heat and moisture throughout the course of the day, material systems can provide passive cooling. This capacity to delay the movement of moisture from outside to inside, known as the time lag factor, allows building materials to regulate and balance moisture and temperature in ambient air.

\section{The vernacular thatch roof model}

Like many vernacular systems, thatch roofs, a fibrous assembly of woven or bundled straw/reed, combines the hygroscopic properties of natural fibres with an overall geometry that directs water and airflow. Though varying regionally in construction materials and techniques, typically a thick waterproof shell (up to 2 metres) is created by layering bundles of watershedding plants. Roofs slopes vary from $45-60$ degrees and roof shapes may be angular or undulating. Water can penetrate the topmost layers of the roof. Straw and reeds have a cellular composition of long, hollow, overlapping parallel fibres that allow for flexibility and surface water repellence.

In this research, the thatch roof is treated as a hierarchical material system. It is programmed with hydrophobic and hydrophilic behaviours at different scales. The roof's surface texture and topology repels water. The bundling and layering of thatch creates a matrix of interstitial air spaces for ventilation and insulation, where variations in density and porosity will control airflow. The cellular structure of straw or reed has the capacity to absorb and release water in balance with the environment. While a thatch roof does not retain large amounts of water, thatch materials with larger diameters have more surface area for water absorption and a greater capacity for moisture storage, creating an increased delay in ambient moisture flows. The thatch roof 
serves as a conceptual model for a thickened, reticulated fabric with functionally graded moisture controls.

\section{Physical Experiments}

Early material studies explore ways to gain a visual understanding of the relationship between fibre, pattern and water capture. These experiments consider how fibre type influences water cohesion and collection, and how the fabric's structure affects water transport. Figure 1 demonstrates the collection of moisture on prototypes knitted with different fibres using a mist spray nozzle. Organic fibres such as wool, which "wicks" moisture absorbing and transporting water - show water droplets on the fabric's surface, while synthetic fibres like nylon thread - which do not absorb but does transport moisture easily - do not collect water droplets, but effectively repels and carries water across its surface.

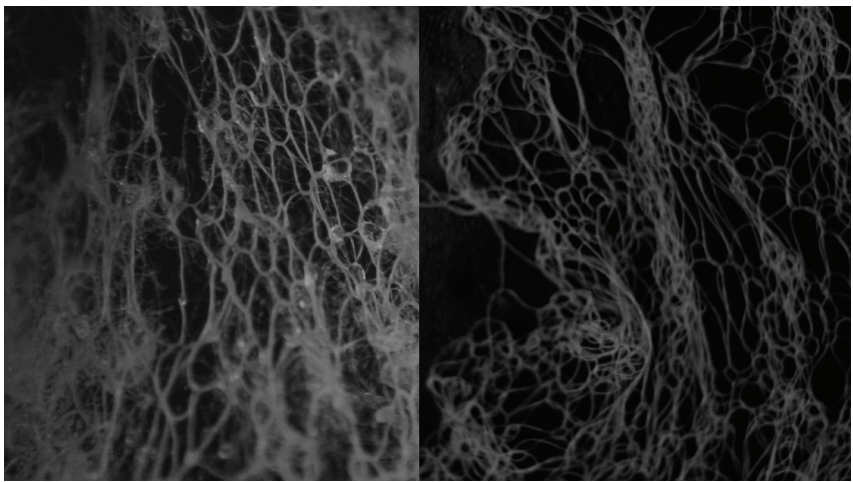

Figure 1: Tests showing knitted prototypes in wool/mohair (right) and nylon (left) fibres. Wool droplets collect and bead while the nylon net simply becomes saturated.

The studies were extended into a larger project, Lemon, which explores a multi-scalar approach to moisture collection and channelling through fabric deformation. Lemon, a fibre and ice installation at MNBAQ, sought to capture the changeable properties of water in a material state of freezing, specifically testing deformed fabrics and the time required for freezing. Tests were conducted using manufactured cotton cheesecloth of a specified density - 28 threads per inch - and a mist-spray nozzle at -23 degrees celcius. Fabrics were deformed to given new curvatures at the material scale and at a 'human' scale. At the scale of the material, by pinching the cheesecloth to variegate the fibre distribution, a reticulated surface was created for improved moisture collection. At a larger scale, by gathering panels of $1 \mathrm{~m} \mathrm{x}$ $2 \mathrm{~m}$ fabric along specified grid lines, curved topologies were generated. Finally, by layering multiple fabrics connected by more porous interstitial layers (which were laser-cut to create porosity), moisture transport could be faciliated across different layers by 'misting' the top layer and watching secondary layers soak through and expand.
These material manipulations offer an (albeit imprecise) way of visualizing water transport on different fibres and patterns, by artificially spraying water across the fabrics, and using freezing to capture the movement of water. Far from addressing more the more complex and subtle phenomenon of time lag across material assemblies for atmospheric moisture balance, the fabrics nevertheless show moisture movement over time.

\section{Digital Tool Development}

Physics simulation in Processing (a java-based programming language) provides access to the dynamic interaction of moisture and material microstructure, topology, and time. By modeling the interaction between water particles and curvature for water repellence, we determine the conditions for a reticulated hydrophobic surface. These conditions are applied to a simulated fabric by applying curvature onto a deformed grid. Further applying branching systems and source/sink flows, an overall topology can be generated that directs water movement across a fabric, while patterned reticulations enhance water flow and retention.

\section{Particle system simulation}

Particle systems have recently been adopted by architectural researchers to provide an elementary means of modeling and generating iterative patterns such as tension-active structures (Ahlquist, 2009). Particle systems simulate the dynamic movement of a group of particles, where each particle carries attributes of mass, location, velocity and acceleration that can influence how the system behaves as a whole. Particles respond to programmed environmental forces at each moment in time which can in turn change where and how they are rendered. A flexible quantity of particles are stored in an array list and retrieved as new actions are applied. Particle systems are used to emulate the behaviour of natural phenomenon with irregular quantities (Shiffman, 2012), and to articulate soft and rigid bodies using interconnected particles.

In this investigation, two types of particle systems are modelled: liquid movement and the simulation of a fabric using a particle spring system where material properties can manipulated and applied as forces. Both systems are modelled in relation to surface curvature as a starting point to determine the interaction between fibre configurations and moisture movement. 


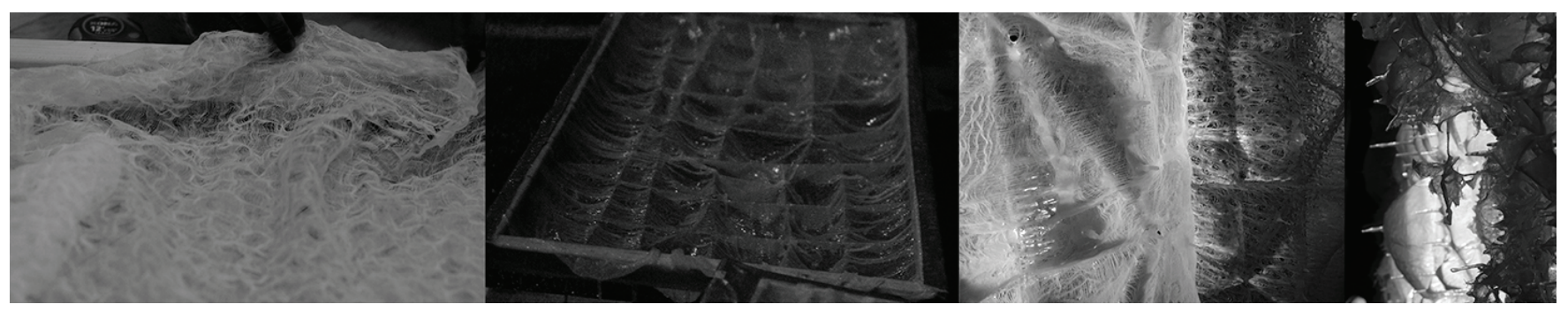

Figure 2: Craft process and results for Lemon. From left to right: (i) Pinching fabric by hand to variegate fabric microstructure. (ii) Deformed "bumps". (iii) Frozen water textures on deformed fabric. (iv) Water capture and freezing on layered fabric with laser-cut connections.

\section{Simulating hydrophobia and hydrophilia}

While sophisticated means of dynamic fluid simulation exist in computer graphics to visualize fluid motion over time, this investigation simply tests the interaction of water molecules with a curved surface in a two-dimensional diagrammatic tool. Liquid movement is encoded as a particle system, with water molecules represented by each particle, programmed to attract, repel, or cohere based on contact angle with one another, and to permeat or collect upon interaction with a curved surface. Iterations of a curved surface, represented in section by a two-dimensional line, is generated using basic trigonometry.

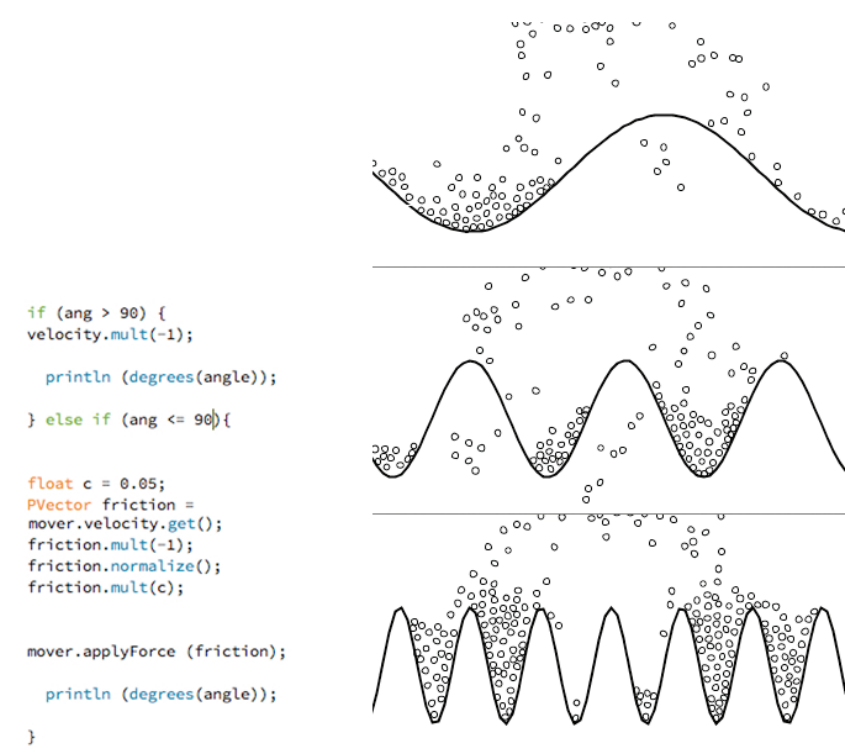

Figure 3: Simulation showing curvature and moisture repellence. Curvature varies by changing increment on sine wave. Water droplet size is exaggerated for clearer graphics.

\section{Fibre configurations for moisture control}

Cloth simulations are modelled using particle spring systems, where particles are connected by a network of springs. Particles carry a scalar attribute of mass and vector attributes of location, velocity, acceleration to track motion, with the location of a particle is given by $f=m a$, where $f$ is the vector sum of all forces. Individual particles are connected by springs calculated using Hooke's law, which states that the force of the spring is directly proportional to the extension of the spring, given by Fspring $=-\mathrm{k} *$ $\mathrm{x}$, where $\mathrm{k}$ is a spring constant that determines the elasticity or rigidity and scales the force applied, and $x$ refers to the displacement of the spring's current length from its rest length. Vector forces oscillating between springs resolve the system to a force equilibrium state, transforming the spring network into a defined geometry. A Verlet Physics integration algorithm based on acceleration and change in position solves the accumulation of vector forces in a spring network, while constraints that specify a fixed distance between connected particles enable the simulation of a soft fabric body. By varying the number of springs, the distance specified for the constraints, and the spring stiffness across the network, different topologies are created.

To generate a fabric, particles are organized into a double array via a nested for-loop, generating a grid with $\mathrm{x}$ and $\mathrm{y}$ coordinates. A basic orthogonal grid of rows and columns can thus represent a basic fabric constructed as successive rows with regular stitch counts, while diagonal springs added to the basic structure helps mimic fabric shear and stiffness. Shifting how the for loop populates or reads variable data in the array provides a means to deform the regular grid to create surface topologies and pattern configurations. These patterns, along with varying material inputs for the springs, allow the material to interact with moisture.

As an array of interconnected particles, the particle spring system illustrates the forces acting upon it and articulates an algorithmic network topology. It is important to note that a network topology is both logical and physical: The arrangement or particles or nodes in the network are defined by pathways of communication and associations within the network (Ahlquist, 2009). This is consistent with mechanical assembly of a fabric, where the form and material behaviour of the fabric is given by the relationship between interlinked elements. 


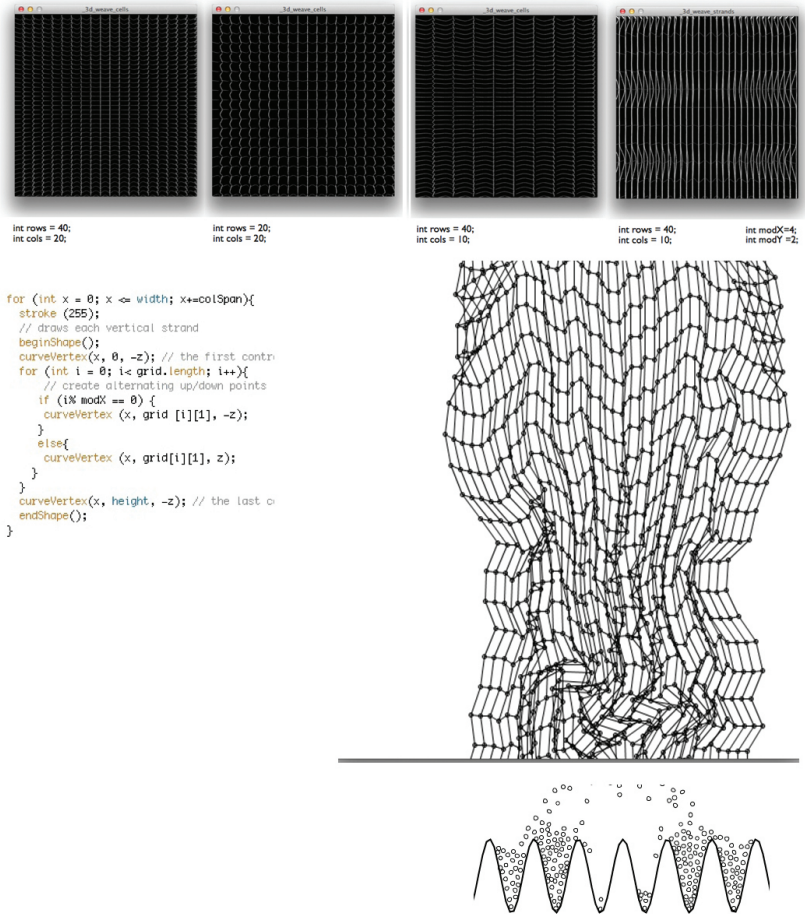

Figure 4: Fabric patterns generated by alogrithmically controlling how fibres are distributed in a grid (top); applying sine curvature to deform a regular particle spring grid.

\section{Conclusion and Further Steps}

This preliminary research explores a method for making watersensitive architectural fabrics based on an examination of the subtle moisture controls in vernacular architecture. It illustrates the synergies between the passive environmental response of vernacular material systems and new design paradigms using simulated environmental force systems and generative algorithms. Through complementary studies in computationally generated fibre configurations and physical experiments, the research develops algorithmic relationships between material microstructure, topology and moisture movement. It explores the potential for fabricating functionally-graded fabrics capable of the subtle moisture controls found in vernacular architecture, and also raises questions about new possibilities and methods to learn from the intelligence of vernacular material craft.

\section{Acknowledgments}

This research project is supervised by Professor Philip Beesley at the University of Waterloo and supported by resources and collaborations with Philip Beesley Architecture Inc. and UWSA F_RMlab (digital media and research.)

\section{References}

Ahlquist, S. (2009). Computational Spring Systems. Architectural Design, 79, 130-133.

Andrasek, A. (2012). Open Synthesis: Towards a Resilient Fabric of Architecture. Log, 25. 45-54.

Chen, Q., Fan, J., Sarkar, M., \& Bal, K. (2011). Plant-Based Biomimetic Branching Structures in Knitted Fabrics for Improved ComfortRelated Properties. Textile Research Journal. Retrieved from http://trj.sagepub.com/content/early/2011/03/25/0040517510397 579

de Beaureceuil, A. \& Lee, F. (2008). Environmental Ornamentation. In Environmental Tectonics: Forming Climatic Change. S. Hardy (Ed.) London: AA Publications.

Eadie, L. \& Ghosh, T. (2011). Biomimicry in textiles: past, present and potential. An overview. Interface: Journal of the Royal Society, 8 59. Retrieved from http://rsif.royalsocietypublishing.org/content/8/59/761.full

Fathy, H. (1986). Natural Energy and Vernacular Architecture. Tokyo: United Nations Press.

Scott, J. (2014). Heirarchy in Knitted Forms. In Proceedings from ACADIA Conference 2013. Toronto: Riverside Architectural Press.

Straube, J. (2006). BSD-138: Moisture and Materials. Building Science Digets. Retrieved from http://www.buildingscience.com/documents/digests/bsd-138moisture-and-

materials/?searchterm=moisture\%20and\%20materials

Shiffman, D. (2012). Particle Systems, The Nature of Code. Retrieved from http://natureofcode.com/book/chapter-4-particle-systems/

Trafton, A. (2006). Beetle Spawns New Material. Retrieved from http://web.mit.edu/newsoffice/2006/beetles-0614.html

Warwick, P. (2005). In Extreme Textiles: Designing for High Performance. M. Mcquaid (Ed.) New York: Princeton Architectural Press. 